\title{
The Relation between AEDs and Liver Disease
}

\author{
Ghaydaa Ahmed Shehata* \\ Professor of Neurology, Assuit University, Egypt
}

*Corresponding author: Ghaydaa Ahmed Shehata, Professor of Neurology, Assuit University, Egypt.

Received Date: December 20,2019

Published Date: January 14, 2020

\begin{abstract}
Nowadays, the relationship between epilepsy and liver disease has risen. The prevalence ranged from $5.5-6.69 \backslash 1000$ in wide epidemiological studies in Egypt (El-Tallawy et al., 2016, El-Tallawy et al., 2013, Farghaly et al., 2013). Antiepileptic drugs (AEDs) are no longer restricted to the treatment of epilepsy. These are widely used in a broad spectrum of psychiatric and neurological disorders. Various liver diseases affect the response to different antiepileptic drugs (AEDs) characteristics to different degrees (Vidaurre et al., 2017) [1]. The liver plays a major role in the metabolism of many of these drugs. Hepatotoxicity is rare, but a real concern when initiating therapy. Likewise, liver disease can adversely affect the biotransformation of some of these drugs. As the disease progresses, the proportion of disrupted liver functions may change, so that acute liver disease may affect AEDs differently than chronic liver failure (Boggs, 2011) [2]. Symptomatic seizures or epilepsy may complicate the course of the hepatic disease to different degrees. Initiating the treatment of antiepileptic therapy in this setting represents a difficult challenge because most AEDs are metabolized by the liver (Vidaurre et al., 2017) [1]. Severe liver disease also affects the binding capacity of (AEDs) to serum proteins, increasing the risk of toxicity. Cirrhosis diminishes total hepatic blood flow but does not impact hepatocyte function until it is severe. Hepatitis may increase hepatic blood flow but diminishes hepatocyte function (Boggs, 2011) [2].
\end{abstract}

\section{Hepatic Biotransformation of AEDs}

Liver disease can affect the metabolism of AEDs in several ways and due to different underlying etiologies. Drug metabolism depends on different factors as hepatic blood flow, albumin binding, and the degree of drug uptake by the hepatocyte (Boggs, 2011) [2]. In addition, the functional integrate of the hepatocytes and finally the patency of the hepatobiliary system. A functional compromise at any level can potentially impair biotransformation, causing parent compounds to accumulate or the generation of active metabolites to be interrupted and affect upon the efficacy of AEDs (Ahmed and Siddiqi, 2006) [3].

The decision to continue or withdraw drug therapy depends on; the underlying pathology, the extent of hepatic insult, the role of the AED in potentially accentuating the insult and finally the risks (status epilepticus) of discontinuing this agent (Vidaurre et al., 2017) [1].

In some instances, it is safe to continue the use the same drug at a lower dose and, in others, it is not safe to continue the drug at all. Impaired biotransformation can lead to an alternative route of metabolism and, the generation of hepatotoxic metabolites, as in Valproic acid (VPA)-induced hepatotoxicity. The Child-Pugh classification for alcoholic liver disease and portal hypertension classifies the liver disease as mild, moderate and severe (ChildPugh Grade A, B, and C, respectively)

This classification considers five parameters: bilirubin; albumin; prothrombin time; and the presence of encephalopathy and ascites. The number of abnormal variables determines the score, with a higher score representing a more severe disease (Ahmed and Siddiqi, 2006) [3].

The Assessment of Liver Functions and Hepatotoxicity During AED Therapy

Liver enzymes can serve as markers of hepatocellular injury or of an obstruction in the bile flows. Although these enzymes are elevated in the liver disease, the elevation can also be secondary to enzyme induction in the absence of hepatic pathology. An elevated partial thromboplastin time (PTT) or decrease in albumin along with elevated liver enzymes is a more specific marker of liver dysfunction. Carbamazepine (CBZ), Phenobarbital (PB) and Phenytoin (PHT) are potent enzyme inducers (Camfield et al., 1989) [4]. On the other hand, Topiramate (TPM) has weak enzymeinducing characteristics. 
A few weeks to a month's therapy with one of the enzyme inducers lead to a modest elevation of (aspartate aminotransferase [AST], alanine aminotransferase [ALT], (alkaline phosphatase [ALP] and gamma-glutamyl transferase [GGT] beacause a less than twofold increase in ALT, AST and ALP are usually insignificant (Arroyo et al., 2005a, Arroyo et al., 2005b) [5,6]. Because ALP can originate from both the liver and bone, an elevated ALP, in the absence of elevated GGT, points to an extrahepatic origin. Hyperammonemia is also a marker of liver disease, and a four to five-fold increase in levels is associated with central nervous system (CNS) manifestations (Guo et al., 2019) [7].

Apart from VPA, AEDs typically do not lead to higher ammonia levels. A two to three-fold elevation in serum ammonia can result during VPA therapy and is usually insignificant. This elevation probably results from a decreased synthesis of mitochondrial acetyl CoA, leading to a decrease in $\mathrm{N}$-acetyl glutamate, an activator of carbamoyl phosphate synthetase (Ma et al., 2019) [8]. A more than two to three-fold increase in liver enzymes during AED therapy should caution the physician of the potential of coexistent liver disease. If next follow-up reveals a progressive increase in the values of the enzymes, investigations for coexistent liver disease are warranted, and may need a switch to an alternative AED. AED-induced liver disease can be a part of a generalized hypersensitivity reaction, as is recognized with CBZ, lamotrigine (LTG), PB and PHT. Cases of hypersensitivity reaction with other AEDs are also reported. Fever, transient skin rash, eosinophilia and lymphadenopathy are associated features (Boggs, 2011, Guo et al., 2019, Lavanchy, 2009, Ren et al., 2019) [2,7,9,10]. Recommendation of dosing and monitoring of AEDs in liver disease (Boggs, 2011) [2] (Table 1 \& 2).

Table 1: Recommendations for usual dosing and monitoring of specific antiepileptic drugs (AEDs) in liver disease . $^{\text {. }}$

\begin{tabular}{|c|c|c|c|}
\hline Drug & Amount of Dosing Reduction & Useful Metabolic Labs to Monitor & Frequency of Labs Examination \\
\hline Barbiturates & $50 \%-75 \%$ & AST, ALT, Coag, & $1-2$ mo \\
\hline Phenytoin & $50 \%-75 \%$ & AST, ALT, Coag,Albumin & $1-2 \mathrm{mo}$ \\
\hline Carbamazepine & $50 \%-75 \%$ & AST, ALT, Coag,Albumin, $\mathrm{Na}+, \mathrm{CBC}$ & $1-2 \mathrm{mo}$ \\
\hline Oxcarbazepine & $25 \%-50 \%$ & AST, ALT,Na+,Cr & $1-3 \mathrm{mo}$ \\
\hline Valproic acid & $25 \%-50 \%$ & AST, ALT,Coag,Albumin, platelets & $1-2 \mathrm{mo}$ \\
\hline Ethosuximide & $25 \%-50 \%$ & AST, ALT,Coags,CBC, platelets & $1-2$ mo \\
\hline Benzodiazepines & $50 \%-75 \%$ & AST, ALT,Coag & $1-2$ mo \\
\hline Lamotrigine & $50 \%-75 \%$ & AST, ALT,Coags,levels & $1-2 \mathrm{mo}$ \\
\hline Gabapentin & Minimal & - & $3-6 \mathrm{mo}$ \\
\hline Pregabalin & Minimal & - & $3-6 m o$ \\
\hline Topiramate & $25 \%-50 \%$ & AST, ALT,Coags & $3-6 m o$ \\
\hline Zonisamide & $25 \%-50 \%$ & AST, ALT,Coags & $2-6 m o$ \\
\hline Levetiracetam & $25 \%-50 \%$ & - & $3-6 \mathrm{mo}$ \\
\hline Tiagabine & $50 \%-75 \%$ & AST, ALT,Coags & $3-6 m o$ \\
\hline Vigabatrin & None & AST, ALT & $1-3 \mathrm{mo}$ \\
\hline Rufinamide & $25 \%-50 \%$ & - & $3-6 m o$ \\
\hline Lacosamide & $25 \%-50 \%$ & AST, ALT,Coags & $3-6 m o$ \\
\hline Felbamate $^{\mathrm{b}}$ & NA & AST, ALT,CBC,differential & $2-4 w k$ \\
\hline
\end{tabular}

aDosing and monitoring for all patients should be individualized. Monitoring of levels may be helpful in some cases

${ }^{\mathrm{b}}$ Agent of last option in liver disease ALT alanine transaminase, AST aspartate transaminase, CBC complete blood count, coags coagulation values

Table 2: Treatment modalities with hepatotoxicity associated with AEDs (Vidaurre et al., 2017).

\begin{tabular}{|c|c|c|}
\hline AEDs & Treatment & Notes \\
\hline Valproic acid & $\begin{array}{l}\text { l-Carnitine } \\
\text { Hemodialysis, } \\
\text { sodium } \\
\text { benzoate phenyl acetate }\end{array}$ & $\begin{array}{l}\text { Doses of } 100 \mathrm{mg} / \mathrm{kg} \text { followed intravenous doses of } 50 \mathrm{mg} / \mathrm{kg} \text { every } 8 \text { hours, to a maximum of } 3 \\
\text { g per dose. } \\
\text { Used for progressive neurological deterioration or ammonia levels greater than } 680 \mu \mathrm{g} / \mathrm{dL} \\
(400 \mu \mathrm{mol} / \mathrm{L})\end{array}$ \\
\hline Carbamazepine & N-Acetylcystein* & $\begin{array}{l}\text { Doses used are } 150 \mathrm{mg} / \mathrm{kg} / \text { hour over } 1 \text { hour, followed by } 12.5 \mathrm{mg} / \mathrm{kg} / \mathrm{hour} \text { for } 4 \text { hours, then } \\
\text { continuous infusions of } 6.25 \mathrm{mg} / \mathrm{kg} / \text { hour for the remaining } 67 \text { hours }\end{array}$ \\
\hline Phenytoin & Steroids N-Acetylcysteine & $\begin{array}{l}\text { Hypersensitivity reactions } \\
\text { Doses used are } 150 \mathrm{mg} / \mathrm{kg} / \mathrm{hour} \text { over } 1 \text { hour, followed by } 12.5 \mathrm{mg} / \mathrm{kg} / \mathrm{hour} \text { for } 4 \text { hours, then } \\
\text { continuous infusions of } \\
6.25 \mathrm{mg} / \mathrm{kg} / \text { hour for the remaining } 67 \text { hours }\end{array}$ \\
\hline
\end{tabular}




\section{Conclusion}

1. Some antiepileptic drugs without (gabapentin, and pregabalin), or with minimal, hepatic metabolism, such as levetiracetam, zonisamide, topiramate, should be used as firstline therapy.

2. Medications undergoing extensive hepatic metabolism, such as valproic acid, carbamazepine, phenytoin, and felbamate should be used as drugs of last resort.

3. 3-We do recommend a baseline test to identify an existing problem.

4. There is no proven value of routine blood testing for monitoring liver functions in asymptomatic patients.

5. The presence of underlying liver disease may require dose adjustment and not necessarily the discontinuation of the medication. Exceptions apply to FBM, VPA and possibly CBZ.

6. In most established cases hepatic toxicity is idiosyncratic or part of a hypersensitivity reaction.

7. Dose dependent hepatotoxicity is rare and usually reversible with prompt discontinuation of the offending agent.

\section{Acknowledgement}

None.

\section{Conflict of Interest}

No conflict of interest.

\section{References}

1. Vidaurre J, Gedela S, Yarosz S (2017) Antiepileptic Drugs and Liver Disease. Pediatr Neurol 77: 23-36.

2. Boggs JG (2011) Seizure management in the setting of hepatic disease. Curr Treat Options Neurol 13: 333-345.

3. Ahmed SN, Siddiqi ZA (2006) Antiepileptic drugs and liver disease. Seizure 15: 156-64

4. P Camfield, C Camfield, J Dooley, K Farrell, P Humphreys, et al. (1989) Routine screening of blood and urine for severe reactions to anticonvulsant drugs in asymptomatic patients is of doubtful value. CMAJ 140: 1303-1305.

5. Arroyo S, Boothman BR, Brodie MJ, Duncan JS, Duncan R, et al. (2005a) A randomised open-label study of tiagabine given two or three times daily in refractory epilepsy. Seizure 14: 81-4.

6. Arroyo S, Dodson WE, Privitera MD, Glauser TA, Naritoku DK, et al. (2005b) Randomized dose-controlled study of topiramate as first-line therapy in epilepsy. Acta Neurol Scand 112: 214-22.

7. Guo HL, Jing X, Sun JY, Hu YH, Xu ZJ, et al. (2019) Valproic Acid and the Liver Injury in Patients with Epilepsy: An Update. Curr Pharm Des 25: 343-351.

8. Ma L, Pan Y, Sun M, Shen H, Zhao L, et al. (2019) Catalase C-262T Polymorphism Is a Risk Factor for Valproic Acid-Induced Abnormal Liver Function in Chinese Patients with Epilepsy. Ther Drug Monit 41: 91-96.

9. Lavanchy D (2009) The global burden of hepatitis C. Liver Int 29 Suppl 1: 74-81.

10. Lavanchy D (2009) The global burden of hepatitis C. Liver Int 29 Suppl 1: 74-81.

11. Ren T, Xiao M, Yang M, Zhao J, Zhang Y, et al. (2019) Reduced Systemic and Brain Exposure with Inhibited Liver Metabolism of Carbamazepine After Its Long-Term Combination Treatment with Piperine for Epilepsy Control in Rats. AAPS J 21: 90. 